Fanum

Sociológico

\section{Forum Sociológico}

Série II

$20 \mid 2010$

A pobreza, pluralidade de olhares e de intervenções

\title{
Les politiques publiques de lutte contre la pauvreté. Variation sur l'approche française
}

\section{Michel Messu}

\section{(2) OpenEdition}

1 Journals

Édition électronique

URL : https://journals.openedition.org/sociologico/152

DOI : 10.4000/sociologico. 152

ISSN : 2182-7427

Éditeur

CICS.NOVA - Centro Interdisciplinar de Ciências Sociais da Universidade Nova de Lisboa

\section{Édition imprimée}

Date de publication : 1 juin 2010

Pagination : $21-28$

ISSN : 0872-8380

\section{Référence électronique}

Michel Messu, « Les politiques publiques de lutte contre la pauvreté. Variation sur l'approche

française », Forum Sociológico [En ligne], 20 | 2010, mis en ligne le 05 septembre 2012, consulté le 29 mars 2022. URL : http://journals.openedition.org/sociologico/152 ; DOI : https://doi.org/10.4000/

sociologico.152

Ce document a été généré automatiquement le 29 mars 2022.

(c) CICS.NOVA 


\title{
Les politiques publiques de lutte contre la pauvreté. Variation sur l'approche française
}

\author{
Michel Messu
}

1 Les sciences sociales se sont souvent donné pour mission de montrer combien les mots que nous utilisons, les notions et les images avec lesquelles nous nous représentons la réalité sociale, voire les concepts à partir desquels nous théorisons, sont des constructions sociales, historiques, contingentes, opérationnelles mais orientées ou déterminées au regard d'intérêts singuliers. Le pauvre et sa pauvreté, l'exclu et son exclusion, le précaire et son "précariat", etc., ont, de longue date, fait l'objet de telles explicitations. Malgré tout, tout aussi régulièrement, les sciences sociales se sont retrouvées en défaut de vigilance à l'endroit de telles constructions. Elles ont trop souvent consacré sur le plan scientifique ou disciplinaire des constructions dont la validité n'était à envisager qu'au sein des enjeux sociaux qui les entouraient. Tout enjeu de définition, on le sait, renvoie à un enjeu social qui peut être uniquement un enjeu de position dans le champ des forces sociales en présence pour s'approprier, d'une manière ou d'une autre, l'objet du litige. Mais l'enjeu de définition peut-être aussi interne aux sciences sociales ${ }^{1}$. C'est au croisement de tous ces enjeux que se situera notre propos. Nous chercherons à montrer comment derrière les variétés des définitions, les controverses conceptuelles et les polémiques disciplinaires se jouent aussi bien des rapports aux situations empiriques à comprendre et donc à la nature du rapport que les sciences sociales entretiennent avec les forces du social qui se retrouvent sur le même terrain.

2 Aussi procéderons-nous en dégageant ce que nous appellerons des paradigmes d'action publique de lutte contre la pauvreté. Paradigmes que nous analyserons tant au regard des conceptions intrinsèques de la pauvreté qu'ils recèlent et des formes d'actions qu'ils induisent. Le moment et la genèse même de leur élaboration seront regardés comme les révélateurs des enjeux théoriques et épistémologiques sur lesquels nous pensons faire porter la singularité française. 


\section{Les paradigmes}

3 A gros traits nous pouvons distinguer trois grandes périodes depuis la Seconde guerre mondiale qui offrent des références théoriques et pragmatiques à trois paradigmes: le paradigme de la "pauvreté-retard" (1950-1970); le paradigme de la "pauvretéexclusion" (1970-1990); le paradigme de la "pauvreté-citoyenne" (1990-2010).

\section{Paradigme 1: la pauvreté-retard}

4 La France des années 60 s'est déjà installée dans la croissance économique, l'accès à un éventail de plus en plus large de biens, le développement du confort et la quasi certitude qu'elle se trouve dans un régime de sécurité. Sécurité politique, l'Europe est en marche; Sécurité économique et sociale, la planification fixe des objectifs qui sont atteints, la sécurité sociale concerne désormais les travailleurs de l'industrie et du commerce, mais aussi le monde agricole et les artisans-commerçants jusqu'alors réticents. Certes la guerre en Algérie fait rage, les "blocs" se regardent et se menacent toujours et pas seulement à coup de course vers la lune... Mais ne pèse guère dans l'idéologie qui s'installe et que l'on appellera par la suite l'idéologie des "30 glorieuses". Celle qui se nourrit des protections renforcées dont jouissent les travailleurs, et d'abord ceux des grandes entreprises du secteur public et para-public et les fonctionnaires et assimilés, qui enregistre les premières vagues de la massification scolaire et culturelle, qui bascule du côté de la consommation et oublie les temps des restrictions et de la rudesse de la vie. C'est la société de consommation, confiante en son avenir de progrès et en l'accroissement de ses capacités qui s'impose comme une évidence.

5 Malgré tout, le scandale rôde. L'hiver 54 et l'appel de l'abbé Pierre en faveur des mal logés; les "poches de pauvreté" qui subsistent ça et là, dans les campagnes notamment autour d'une agriculture familiale jugée archaïque; les bidonvilles qui fleurissent en périphérie des plus grands centres urbains à mesure que se résorbe l'habitat insalubre des centres-villes et que s'installe les grandes entreprises consommatrices de main d'œuvre peu qualifiée; des fractions du peuple des villes et des villages subsistent tant bien que mal de petits métiers ou d'expédients et ne peuvent réaliser les rêves consuméristes des populations plus aisées; etc. Le scandale, c'est la persistance de groupes de la population qui restent en situation de pauvreté, qui n'ont pu prendre en marche le train de la croissance et du confort, qui se révèlent donc inadaptés à la nouvelle société. Cette pauvreté-là est pensée comme résiduelle, comme un retard au regard des progrès accomplis par les autres.

\section{Paradigme 2: la pauvreté-exclusion}

6 Après le premier choc pétrolier et les retournements locaux de conjoncture économique, la confiance dans l'avenir s'estompe. C'est la montée des inquiétudes, comme le dira le CRÉDOC. D'abord l'inquiétude de voir son emploi menacé, du moins dans les secteurs les plus fragiles face à l'économie-monde qui se dessine et multiplie les délocalisations et les concentrations. La perte de son emploi, les difficultés pour le retrouver ou y accéder sont bientôt perçues comme des risques d'exclusion sociale. 
Donc de perte du statut antérieurement acquis et de ce qui pouvait lui être associé, notamment en termes de consommation de biens et de services.

7 La figure du pauvre prend dès lors les traits d'un "exclu", d'abord de l'emploi, puis et au fur et à mesure du succès de la notion d'exclusion dans l'opinion publique de tout segment social d'intégration: logement, école, loisirs, santé, etc. La pauvreté, désormais se pense dans les termes d'un manque provoqué par une perte ou un obstacle mis à l'obtention de ce qui est convoité.

\section{Paradigme 3: la pauvreté-citoyenneté}

De l'exclusion, la pauvreté va se faire "citoyenne" lorsque la trajectoire du regard va s'inverser et envisager non plus l'exclusion du pauvre mais son inclusion dans des segments d'intégration sociale, ou, du moins, pensés comme des voies favorables à des formes d'intégration sociale jugées plus satisfaisantes. Il ne s'agit plus de recouvrer ce qui a été perdu mais de s'adapter aux conditions nouvelles nées de l'approfondissement de la mondialisation des échanges, en particulier de s'adapter aux rythmes des changements qu'elle impose. La thématique de la flexibilité, de la réactivité, tend à s'imposer dans bien des domaines de la vie sociale. Elle s'impose aussi dans la perception et le traitement du pauvre.

Du coup, il est de sa responsabilité de pouvoir se rendre "flexible", de s'engager dans des processus de mise en mouvement, de saisir les opportunités, bref, d'être réactif. Tout cela et tout à la fois au nom de sa citoyenneté intrinsèque, avérée ou promise, et pour la maintenir ou la constituer. Le pauvre est un citoyen, titulaire de droits référencés aux Droits de l'Homme, promis à connaître une vie digne et, à ce titre, tenu à des obligations de participation, d'engagement dans des processus d'inclusion sociale. C'est là sa responsabilité individuelle, fût-il profondément démuni.

\section{Les conceptions}

Chacun de ces paradigmes renvoie, plus ou moins explicitement, à des conceptions spécifiques de la pauvreté. Ces conceptions sont elles-mêmes articulées aux représentations tant de l'époque historique - l'air du temps, si l'on veut -, que des impératifs politiques, sociaux ou moraux qui vont caractériser la dite époque et qui deviennent autant d'objectifs, de vœux, d'espoirs, etc. Et, bien qu'elles ne soient pas strictement partagées par tous, ces représentations vont dominer l'époque en ce qu'elles fournissent ce fond commun de conception, ce substrat théorico-idéologique, sur lesquels s'édifiera l'action publique, étatique comme "privée".

\section{Paradigme 1: la pauvreté-retard}

11 La conception dominante ici est celle du retard culturel. Est pauvre, ou plus exactement, est resté pauvre celui qui n'a pas su, pas pu, pas voulu... prendre le train de la croissance, du développement et de la modernité. L'heure est en effet, en France pour le moins, aux changements structurels. Sur le plan économique, c'est la relance, la croissance, l'augmentation sensible du pouvoir d'achat, etc. Sur le plan économique et social, c'est la planification qui entend maitriser les orientations du développement. 
Sur le plan social et géographique, la France bascule de la ruralité vers l'urbanité, la physionomie de ses villes se transforme, les grands ensembles surgissent, les grands centres d'achats à l'américaine se multiplient, les communications s'améliorent et s'accélèrent, mais aussi la paysannerie familiale, le petit commerce et l'artisanat rural sont dépréciés et en voie de perdition. Bref, la "société de consommation" s'installe et disqualifie ceux qui ne s'y inscrivent pas. Ils ont été dépassés et sont restés sur le bord du chemin du progrès social. Ce sont d'abord des retardataires. Parfois parce qu'ils sont traditionnalistes, d'autres fois parce qu'ils ne disposaient pas des ressources ou des ressorts nécessaires, dans tous les cas parce qu'ils n'ont su, pu ou voulu s'inscrire dans le mouvement de modernisation, de progrès de ce qui se présente comme la "nouvelle société". La pauvreté est ainsi pensée comme un retard au regard du mouvement général, comme un écart à l'endroit de ce qui affecte la société dans sa globalité.

\section{Paradigme 2: la pauvreté-exclusion}

Il en va tout autrement dans les années 70-80 quand la "crise de l'emploi" s'installe, que ceux-là mêmes qui avaient fait le pari de la modernité, qui s'étaient engagés dans la consommation et l'endettement, qui croyaient dans les sécurités qui s'étaient mises en place..., que ceux-là donc se trouvent menacés et en quelque sorte rejetés du chemin du progrès continu. Cette fois la pauvreté n'apparaît plus comme une sorte de conséquence d'un retard pris vis-à-vis du mouvement progressif de la société. Cette fois, c'est la société qui paraît régressive, elle inverse le mouvement de progrès social et l'assurance de sécurité. Du coup, ceux qui subissent les effets de cette inversion prennent les traits d' "exclus", de déclassés, de relégués. Ils sont en quelque sorte bannis de ce qui reste les attentes à l'endroit de l'histoire de nos sociétés. Celles sont toujours perçues comme des sociétés de progrès, de développement et d'accroissement des richesses.

Et de fait, les richesses s'accumulent, mais selon une nouvelle logique que l'on nommera "globalisation" ou "mondialisation". C'est d'ailleurs dans cette période que s'accélèrent des dépenses sociales de l'État et que se mettent en place de nouvelles formules de protection sociale. Formules d'assurance d'assistance, visant une forme de solvabilisation de ceux qui versent dans des situations de pauvreté au nom de la dignité humaine qui leur est fondamentalement reconnue. Exemplaire de ce point de vue est l'adoption en décembre 1988 de la loi sur le "Revenu minimum d'insertion" dont l'objectif affiché est de favoriser le retour dans des segments de l'intégration sociale (emploi, santé, formation...).

La pauvreté, que l'on déclinera d'ailleurs en degrés, avec donc des situations d' "extrême pauvreté" ou de "très grande pauvreté", quand d'autres ne seraient qu'ordinaires sous un "seuil de pauvreté" monétaire qui devient la toise d'appréciation de la pauvreté elle-même, nonobstant les critiques théoriques et empiriques que ce dernier pouvait recevoir de la part des chercheurs ou des institutions et des militants associatifs. La pauvreté d'exclusion, prétendra-t-on à ce moment, serait susceptible de toucher "un français sur deux". C'est dire combien la définition du pauvre s'est substantiellement transformée. D'un retard d'intégration dans la modernité, elle est devenue le résultat d'une mise à l'écart ou d'un maintien à l'écart du progrès continué de nos sociétés de richesses. Car c'est le procès même d'accumulation de la richesse dans ces sociétés qui produit ces formes d'exclusion de certains segments d'intégration 
sociale et engendre la formation de formes d'inclusion dans de nouveaux segments explicitement désignés comme segments d'inclusion sociale. D'où, tous les enjeux politiques et sociaux sur le tracé des lignes de partage et les frontières entre inclusion dans la société d'abondance et de sécurité et l'inclusion dans les dispositifs de sécurisation et de subsistance.

\section{Paradigme 3: la pauvreté-citoyenneté} pauvreté, que du côté des pauvres eux-mêmes, l'heure en soit à une vision de la pauvreté comme défaut de citoyenneté sociale. Lequel défaut est pensé comme induisant une moindre citoyenneté politique. Autrement dit, le pauvre, même lorsqu'il se trouve encore appréhendé dans le registre du moindre avoir, ne saurait être maintenu dans celui du moindre être. de l'assistance de la collectivité. Il marquera, en retour, sa solidarité avec le collectif en s'inscrivant dans des démarches actives d'inclusion dans les segments de l'intégration sociale.

\section{Les politiques publiques}

Ce qui caractérise la situation du pauvre dans nos sociétés développées, démocratiques et de protection est que celui-ci reste l'objet de la sollicitude des pouvoirs publics. Quelle que soit la conception sous laquelle il va être appréhendé, quel que soit le paradigme dans lequel il sera inclus, les pouvoirs publics restent l'instance qui va décider de son sort prochain. Sous la forme d'une mise en œuvre d'une politique publique, les pouvoirs publics vont être mobilisés pour répondre à la situation du pauvre. Mieux, par leur intervention ils vont le constituer comme pauvre à prendre à charge, comme pauvre à intégrer dans la collectivité.

\section{Paradigme 1: la pauvreté-retard}

Quand le pauvre se présente comme un laissé pour compte de la croissance, du progrès social et de l'accès à la modernité, les pouvoirs publics vont imaginer une politique d'intégration basée sur l'éducation, l'accompagnement et l'acquisition des "bonnes pratiques" de la société de consommation. C'est en effet en termes d'interventions correctrices, de réadaptions, de conseils et d'accompagnement dans le changement que 
seront conçues les politiques qui, dans les années 70, vont chercher à éradiquer les "poches de pauvreté" qui subsistent dans la "nouvelle société" - pour reprendre une formule alors en vogue et forgée par le Premier ministre Chaban-Delmas et ses conseillers Jacques Delors et Simon Nora. Il s'agit d'abord de donner à l'État, au-delà de ses fonctions régaliennes traditionnelles, un rôle de premier plan dans le changement social jusques et y compris dans l'absorption de ceux qui ont été tenus à l'écart de ses bénéfices. Réadapter les "exclus", dira ensuite René Lenoir, futur secrétaire de l'Action sociale du Président Giscard d'Estaing.

Ainsi envisagée, la politique publique reviendra à doter les "pauvres" d'alors de moyens pour intégrer la société moderne, de progrès et de sécurité. Le logement standardisé et de confort moderne - les Habitations à loyer modéré (HLM) construites en grand nombre pour résorber l'habitat insalubre des couches sociales les moins fortunées -; l'accompagnement socioculturel en direction des populations urbaines restées en marge des nouveaux standards de vie; le suivi et le conseil familial auprès des familles les moins adaptées aux nouvelles conditions de la vie sociale urbaine et consumériste; la prévention de la délinquance juvénile, la prévention des risques sanitaires, la démocratisation culturelle; etc., sont donnés pour les moyens les plus sûrs pour combattre la pauvreté-retard.

21 C'est dans cette période que les professions du "social" vont croître de manière quasi exponentielle - le célèbre numéro de 1972 de la revue Esprit en témoignera tout en y décelant, dans une optique toute foucaldienne, une orientation vers une disciplinarisation des consciences: les pauvres y perdraient leur âme en quelque sorte. C'est dans cette même période que les équipements sociaux destinés aux populations les moins bien dotées socialement et culturellement vont être décrits comme des instruments de leur "normalisation", donc d'atténuation de leur pouvoir subversif. C'était là reconnaître que les politiques publiques mises en œuvre avaient un effet réel sur les situations des plus démunis.

\section{Paradigme 2: la pauvreté-exclusion}

Les crises politiques et économiques des années 80 vont entraîner une rupture et une reconversion des politiques publiques. Celles-ci vont résolument s'engager dans une forme de ciblage et en même temps d'élargissement des populations à traiter comme pauvres. L'accent mis sur la hauteur des allocations à attribuer aux groupes de population jugés en difficulté ou défavorisés eu égard à leur situation via la multiplication de ce que l'on va appeler les minima sociaux, rend compte de ce que les politiques publiques entendent désormais combattre la pauvreté par la solvabilisation de ceux qui connaissent des manques ou des pertes de leur pouvoir d'intégration sociale. La monoparentalité, le handicap, la vieillesse mal pensionnée, la perte de l'emploi rémunérateur, etc., deviennent les "cibles" de mesures et de dispositifs spécifiés censés les prémunir contre des formes de déchéance sociale, contre le basculement dans une forme d'exclusion radicale. Le RMI, déjà évoqué, mais aussi l'API (Allocation de parent isolé), l'AAH (Allocation adulte handicapé), plus tard la CMU (Couverture maladie universelle), etc., se proposent, par la hauteur de la prestation apportée, de maintenir ces groupes de population spéifiés dans un état de dignité sociale que ne pouvaient fournir les aides et autres secours d'antan. 

progrès social, elle se donne comme un risque encouru par, si ce n'est encore le plus
grand nombre, du moins un nombre conséquent d'individus en mal d'intégration avérée dans le collectif social. Partant, elle assigne aux politiques publiques une fonction intégrative active qu'elles seront bien en peine de réaliser. D'autant qu'avec la crise de l'emploi qui s'est propagée dans certains secteurs économiques, la visée d'inclusion ou de réinsertion dans l'emploi des chômeurs de longue durée, ceux qui avaient versé dans les dispositifs de lutte contre la pauvreté, s'est largement trouvée irréalisable. Le stock des sans emploi qui constituent la masse des pauvres décomptés s'alourdit et se dénombre à un niveau qui semblait ne pouvoir être jamais atteint. La lutte contre les exclusions, devenue pourtant, avec la loi du 29 juillet 1998, un impératif politique majeur, fait l'aveu de son impuissance à éradiquer la pauvreté-exclusion. Même son de cloche du côté de la politique de la ville, elle aussi engagée et poursuivie sur une échelle toujours plus large pour combattre la pauvreté-exclusion à partir du territoire. En sommes, si les pauvres sont mieux protégés, en tant que "pauvres", ils restent toujours pauvres - et peut-être même de plus en plus appauvris, notamment dans certaines zones urbaines - et la pauvreté-exclusion subsiste.

\section{Paradigme 3: la pauvreté-citoyenneté}

La décennie 2000-2010 accuse un nouveau changement d'orientation des politiques publiques. Elle s'affiche de plus en plus comme une politique de gestion des situations de pauvreté. Celle-ci s'impose d'autant plus qu'aux pauvres jusqu'alors recensés et répertoriés par leurs manques (emploi, domicile, santé...), s'ajoutent désormais ceux que l'on désignent du vocable de "travailleurs pauvres". Il s'agit de personnes qui, bien qu'ayant accès à l'emploi, n'en tirent que de faibles revenus ou ne les tirent que de manière épisodique. Ce faisant, elles constituent une nouvelle figure de la pauvreté. Une nouvelle notion va d'ailleurs s'imposer pour le dire: le "précariat".

Aussi, toujours au nom de la dignité qui leur est reconnue, et dans le but de les maintenir dans les segments reconnus de l'intégration sociale, les politiques publiques vont les incorporer dans leurs cibles et leurs logiques d'action. Le RSA (Revenu de solidarité active), entré en vigueur le $1^{\mathrm{er}}$ juin 2009, rassemble dans une même intention d'inclusion sociale ceux qui, jusqu'alors concernés ou non par diverses mesures et allocations, étaient dans des situations opposées au regard de l'emploi. L'exclusion/

/inclusion dans l'emploi n'est plus le marqueur des situations de pauvreté. La cible s'est encore élargie et incorpore des fractions de population laborieuse antérieurement perçues comme protégées et non concernées par les politiques publiques d'insertion et de solvabilisation.

Mais l'intervention publique se fait cette fois de manière plus contraignante et la charge de la contrainte pèse plus fortement sur le bénéficiaire de l'aide qu'elle ne l'était 
antérieurement. Et cela, quand les moyens de l'État - surtout depuis la décentralisation de la majeure partie des compétences d'action sociale - se trouvent moins assurés et plus inégalement répartis. C'est la logique de l'activation du bénéficiaire, de sa responsabilisation au titre de sa citoyenneté. L'assistance passive du pauvre est combattue pour sa trop forte propension à favoriser une sorte de parasitisme social. Le pauvre est donc sommé de sortir de sa condition d' "exclu" et de rejoindre les rangs des citoyens responsables, actifs et, donc, contributifs au bon agencement social.

Cette responsabilisation de l'individu pauvre se fait sur un fond de durcissement du contrôle de la part de l'action publique. La solvabilisation du pauvre est maintenue à une hauteur comparable à celle accordée dans la période précédente, parfois elle est même renforcée (RSA, par exemple). Mais elle n'est assurée que conditionnellement, sous condition d'activation notamment. Pour autant, les "filets de sécurité", comme on les a souvent appelés, mis en place dans la période précédente, restent opératoires, même s'ils tendent à s'éroder.

\section{Les enjeux théoriques}

29 Au-delà de ce qui se joue sur la scène politique et sociale en direction des pauvres et des catégories d'appréhension et d'action qui leur sont appliquées, il se profile un certain nombre d'enjeux théoriques auxquels la sociologie se révèle plus ou moins sensible. Certains n'ont rien de bien nouveau et renvoient à ce que Georg Simmel appelait la "réaction sociale" constitutive du pauvre. D'autres semblent plutôt receler les espoirs déçus de voir triompher une société non pas, sûrement, sans pauvre mais une société à même d'égaliser les conditions sociales au point de ne conserver qu'un taux marginal de pauvres. D'autres encore vont s'attaquer au sens théorique qu'il convient de trouver derrière les transformations, glissements, mutations que nous venons d'évoquer. Retenons et reprenons ces trois types d'enjeu théorique.

\section{La réaction sociale constitutive du pauvre}

Malgré les nombreux travaux d'historiens, de politistes, de sociologues, etc., la tendance à penser substantiellement la pauvreté garde des adeptes. Bien sûr c'est plutôt du côté des associations caritatives et des ONG "sociales" que l'on rencontre la tendance. La quête de la parole du pauvre comme parole de vérité de la pauvreté, en est une manifestation bien connue. Car, par delà l'expérience vécue par les pauvres - tout à fait incontestable celle-là -, se trouve recherché un discours singulier, une intrinsèque vérité de ce que serait l'état de pauvreté, ce que les sciences sociales n'ont su produire. La critique des indicateurs et autres instruments de mesure de la pauvreté prend souvent cette allure. On y dénonce leur impuissance à saisir au plus près la "vraie" pauvreté, leur faible capacité à circonscrire ceux qui méritent vraiment d'être secourus, leur formalisme de construction, etc., comme s'il s'agissait de révéler à la surface du monde social une pauvreté recelée dans ses entrailles, et de le faire le plus exactement possible.

31 C'est là méconnaître la leçon simmelienne de la "réaction sociale" qui constitue le pauvre. Pour Simmel en effet, "le but de l'assistance aux pauvres est d'atténuer les manifestations les plus extrêmes de la différenciation sociale, afin que la structure sociale continue à se fonder sur cette différenciation [...] Il n'y a donc aucune raison 
d'aider le pauvre plus que ne le demande le maintien du statu quo social" (Simmel, 1971). Autrement dit, pour le sociologue berlinois, l'action sociale de lutte contre la pauvreté est commandée par une téléologie sociale: celle de la préservation de la collectivité et du statu quo ante qui la caractérise. La téléologie sociale de l'assistance aux pauvres explique d'ailleurs pourquoi, toujours selon Simmel, le droit à l'assistance n'est pas un droit du pauvre en tant que tel, mais le devoir de la collectivité de procéder au maintien de la cohésion sociale. L'assistance au pauvre est fonctionnelle et la fin qu'elle tend à atteindre est purement sociale, abstraction faite des "pauvres concrets", si l'on peut dire, vecteurs individuels de l'action publique. Simmel ramassera son propos en une formule: l'assistance aux pauvres n'est autre chose que l' "application de moyens publics pour des fins publiques" (idem).

N'est-ce pas ce que nous venons de voir en déclinant les divers moments, paradigmes et modes d'action publique qui ont présidé, en France depuis le second conflit mondial, au repérage et à la prise en charge des pauvres. Pauvreté-retard, pauvreté-exclusion, pauvreté-citoyenneté, désignent bien une finalité cohésive on ne peut plus relative à la perception que la collectivité, via ses forces d'imposition politique, se fait d'elle-même. C'est bien pourquoi, tel Sisiphe, les acteurs de la lutte contre la pauvreté n'auront de cesse de poursuivre leur action, de produire des catégories d'éligibilité, de définir des objectifs d'intégration.

\section{L'impensable contradiction sociale}

L'histoire récente de la lutte contre la pauvreté semble construite sur un malentendu théorique. La pauvreté dans les pays riches, comme l'avait titré en son temps Lionel Stoléru ${ }^{2}$, a été pensée comme une sorte d'oxymore social. Que les acteurs de la lutte contre la pauvreté en aient fait un argument idéologique et politique, c'est de bonne guerre, peut-on dire. C'est même un argument judicieux pour provoquer la "réaction sociale" dont nous entretenait Simmel. Une société riche qui n'allège pas la misère des siens, qui ne soutient pas ceux qui subissent un sort contraire, qui ne favorise pas l'égalisation des conditions d'existence sociale, s'écarte du modèle démocratique conçu par les Lumières, soutenu par Tocqueville et théorisé par les solidaristes du XIX ${ }^{e}$ siècle. C'est aussi parce que ce modèle se présente toujours comme l'horizon de nos sociétés que la pauvreté se trouve aujourd'hui subsumée sous la catégorie analytique des droits humains et la responsabilité conjointe des pouvoirs publics et des individus sociaux ${ }^{3}$.

Maintenant, l'étude sociologique ne peut prendre cet objectif politique pour point de vue analytique. Peut-être pourra-t-elle contribuer indirectement à sa réalisation, mais son mobile ne peut s'y réduire. Il lui revient, comme s'y était employé Simmel, de tenter de saisir la signification théorique sociologique, de la "production" sociale du pauvre, de sa "construction" comme enjeu de lutte sociale et politique et donc de sa signification au regard de l'agencement de nos sociétés. Ce qui reste, pour le moins, un des objets d'étude de la sociologie générale.

Du coup, l'objet sociologique "pauvreté" ne peut seulement être envisagé du point de vue de ses enjeux sociaux, il convient de l'intégrer dans une visée de compréhension du mode de fonctionnement et d'existence de nos sociétés. Et donc de l'envisager, si l'on s'en tient à l'optique simmelienne, comme une construction sociale constitutive du social lui-même. On l'a dit, les définitions du pauvre qui se sont succédées historiquement réfléchissent des enjeux de rapports de force entre divers acteurs et 
divers intérêts sociaux et politiques, mais aussi des représentations de la société à produire et reproduire. Et les mesures de politique publique engagées en direction du pauvre ainsi constitué vont produire des effets sur le reste de la société et pas seulement sur lesdits pauvres. Si l'un des enjeux concernant la sollicitude de la collectivité à l'endroit des pauvres qui lui sont coextensifs peut s'énoncer en termes de justice sociale, cette approche n'épuise pas encore le registre sur lequel peut s'inscrire l'analyse sociologique. Relève aussi de ce dernier la compréhension des processus de production des pauvres eux-mêmes, et au-delà de leur seule désignation nominale comme nous l'avons fait plus haut. Dès lors, la question de la production de la pauvreté dans les pays riches peut se formuler en d'autres termes que celui d'un oxymore social. Elle réclame que l'on pense dialectiquement pauvreté et richesse, engendrement du pauvre par le riche et le riche par le pauvre, si l'on peut ainsi s'exprimer. Et cela dans les conditions historiques concrètes que sont les nôtres. Ou encore de penser dialectiquement, mesures de réduction ou d'atténuation de la pauvreté et leurs effets en termes de maintien voire d'extension de celle-ci. Bref, de penser la pauvreté comme une production continuée d'une société qui s'emploie à l'éradiquer.

\section{L'assurance d'assistance}

Dans ce cadre enfin, il revient également à la sociologie de comprendre les logiques des dispositifs qui ont sous-tendu et sous-tendent aujourd'hui les politiques de lutte contre la pauvreté. En France, les historiens, les politistes ont su le faire pour montrer les liens entre les formes de prise en charge du pauvre et vision chrétienne du monde et de la vie sociale, ou encore conception d'un ordre social républicain. Plus récemment le sociologue Robert Castel s'est employé à établir la relation étroite qu'il y a eu entre protection du travailleur et approfondissement du salariat ${ }^{4}$. Il conviendrait sûrement de poursuivre l'analyse de la relation entre mondialisation sous l'égide du néolibéralisme économique et extension d'une pauvreté-citoyenne. Mais cette fois encore, en pensant la relation non pas d'abord dans les termes d'une simple régression au regard de ce qui s'était imposé antérieurement, même si, ici ou là, la régression se trouve avérée, mais également dans les termes d'une reconfiguration plus large des moyens et objectifs de l'action collective.

37 C'est dire que la sociologie gagnerait en compréhension des phénomènes contemporains de pauvreté en forgeant ses propres concepts et cadres d'interprétation. On l'a dit, il aurait été plus judicieux d'analyser les formes de l'exclusion sociale sur un fond d'inclusion sociale élargie ${ }^{5}$. D'envisager la précarité du salarié d'aujourd'hui dans des processus d'intégration renforcée du citoyen. Ou encore de percevoir dans l'hybridation des dispositifs une forme de redistribution des compétences tant du côté de la puissance publique que du côté de la "société civile", comme elle est aujourd'hui désignée, ou encore du marché. Laquelle hybridation s'accompagne d'une redéfinition des compétences du pauvre lui-même. Ce qui provoque aussi la variation de la figure du pauvre et sa participation diversifiée à l'ordre social.

38 Simmel, quand tu nous tiens! 


\section{BIBLIOGRAPHIE}

CASTEL, R. (1995), Les Métamorphoses de la question sociale, une chronique du salariat, Paris, Fayard.

MESSU, M. (1997), “L’Exclusion: une catégorisation sans objet”, Genèses, 27, pp. 147-161.

MESSU, M. (2003), La pauvreté cachée, une analyse bachelardienne du concept de pauvreté, La Tour d'Aigues, Éditions de l'Aube.

SIMMEL, G. (1971), “The poor”, in D. N. Levine, Georg Simmel: On Individuality and Social Forms, University of Chicago Press.

STOLÉRU, L. (1974), Vaincre la pauvreté dans les pays riches, Paris, Flammarion.

\section{NOTES}

1. Je me permets de renvoyer à mon ouvrage: MESSU, Michel (2003), La pauvreté cachée, une analyse bachelardienne du concept de pauvreté, La Tour d'Aigues, Éditions de l'Aube.

2. STOLÉRU, Lionel (1974), Vaincre la pauvreté dans les pays riches, Paris, Flammarion.

3. C'est ce à quoi participent nombre d'ONG et de Mouvements de pression politique, tels ATDQuart Monde, L'Armée du Salut, Caritas, etc.

4. CASTEL, Robert (1995), Les Métamorphoses de la question sociale, une chronique du salariat, Paris, Fayard.

5. Voir MESSU, Michel (1997), “L'Exclusion: une catégorisation sans objet”, Genèses, 27, pp. 147-161.

\section{RÉSUMÉS}

La France est riche d'une tradition d'études, de recherches en sociologie et de politiques publiques sur la pauvreté. L'article propose de dégager les paradigmes d'action publique de lutte contre la pauvreté qui ont scandé l'histoire des 60 dernières années en France: la «pauvretéretard»; la «pauvreté-exclusion»; la «pauvreté-citoyenne». Chacun de ces paradigmes est porteur d'une conception de la pauvreté qui oriente l'action publique. Or, ces conceptions sont aussi des enjeux théoriques pour la sociologie qui envisage les pauvres dans leur rapport à la société dans laquelle ils se trouvent. Trois enjeux sont ici dégagés: «La réaction sociale constitutive du pauvre»; «L'impensable contradiction sociale» et «L'assurance d'assistance».

A França tem uma rica tradição de estudos sociológicos e investigação, bem como políticas públicas relativas à pobreza. 0 artigo visa mostrar que paradigmas têm afectado a acção pública contra a pobreza nos últimos 60 anos em França: “pobreza-atraso", “pobreza-exclusão”, e "pobreza-cidadania". Cada um desses paradigmas transmite uma concepção de pobreza que orienta a acção pública. Ora, essas concepções também representam questões teóricas para a sociologia que observa os pobres na sua relação com a sociedade em que vivem. Três questões 
teóricas são aqui escalpelizadas: "a reacção social constitutiva do pobre”, "a impensável contradição social" e "a segurança de assistência".

France has a rich tradition of sociological studies and research as well as public policies regarding poverty. The article aims to show what paradigms have affected public action against poverty in the past 60 years in France: "poverty-delay», "poverty-exclusion», and "povertycitizenship». Each of these paradigms conveys a conception of poverty that directs public action. Nevertheless, these conceptions also represent theoretical issues for the Sociology that observes the poor in their relationship with the society in which they live. Three theoretical stakes are brought out here: «the social reaction constitutive of the poor», «the unthinkable social contradiction» and «the security of the assistance».

\section{INDEX}

Keywords : poverty, paradigms, public policies, epistemology, France

Mots-clés : pauvreté, paradigmes, politiques publiques, épistémologie, France

Palavras-chave : pobreza, paradigmas, políticas públicas, epistemologia, França

\section{AUTEUR}

\section{MICHEL MESSU}

Université de Nantes \& GEPECS Université Paris-Descartes michel.messu@wanadoo.fr 